\title{
Improved Formulas for Complete and Partial Summation of Certain Series
}

\section{By Herbert E. Salzer and Genevieve M. Kimbro}

\begin{abstract}
In two previous articles one of the authors gave formulas, with numerous examples, for summing a series either to infinity (complete) or up to a certain number $n$ of terms (partial) by considering the sum of the first $j$ terms $S_{j}$, or some suitable modification $S_{j}^{\prime}$, closely related to $S_{j}$, as a polynomial in $1 / j$. Either $S_{\infty}$ or $S_{n}$ was found by $m$-point Lagrangian extrapolation from $S_{j_{0}}, S_{j_{0}-1}, \cdots$, $S_{j_{0}-m+1}$ to $1 / j=0$ or $1 / j=1 / n$ respectively. This present paper furnishes more accurate $m$-point formulas for sums (or sequences) $S_{j}$ which behave as even functions of $1 / j$. Tables of Lagrangian extrapolation coefficients in the variable $1 / j^{2}$ are given for: complete summation, $m=2(1) 7, j_{0}=10$, exactly and $20 \mathrm{D}, m=11$, $j_{0}=20,30 \mathrm{D}$; partial summation, $m=7, j_{0}=10, n=11(1) 25(5) 100,200,500$, 1000 , exactly. Applications are made to calculating $\pi$ or the semi-perimeters of many-sided regular polygons, Euler's constant,
\end{abstract}

$$
1+\sum_{r=1}^{j}\left\{\frac{-1}{(4 r-1)^{2}}+\frac{1}{(4 r+1)^{2}}\right\}=1-\frac{1}{3^{2}}+\frac{1}{5^{2}}-\cdots \text { for } j=\infty
$$

(Catalan's constant), calculation of a definite integral as the limit of a suitably chosen sequence, determining later zeros of $J_{\nu}(x)$ from earlier zeros for suitable $\nu$, etc. A useful device in many cases involving sums of odd functions, is to replace $S_{j}$ by a trapezoidal-type $S_{j}^{\prime}$ which is seen, from the Euler-Maclaurin formula, to be formally a series in $1 / j^{2}$. In almost every example, comparison with the earlier $(1 / j)$-extrapolation showed these present formulas, for $\tau$ points, to improve results by anywhere from around 4 to 9 places.

1. Introduction. In two earlier papers, $[1,2]$, one of the authors gave tables for both complete summation (all terms, to infinity) and partial summation (up to a certain number of terms) of certain kinds of slowly convergent series. In the case of partial summation, divergent series were also included, prorided that a suitable auxiliary series could be found of the desired slowly convergent type and simply related to the original divergent series. The essential idea in both cases is to regard the sequence $S_{j}$, the sum of the first $j$ terms of the series, as the values for $x=1 j$ of an interpolable function $S(x)$ to which the slight extrapolation from specified $S_{j}$, to $j=\infty(x=0)$ or to $j=k(x=1 / k), k>j_{0}$ where $S_{j_{0}}$ is the last specified $S_{j}$, yields good accuracy. The approximating formula for $S(x)$ was an $m$-point Lagrange polynomial of the $(m-1)$ th degree in $x$ which at $x=1 / j$ assumes the prescribed value $S_{j}$, for the last $m$ values of $j$ ending at $j_{0}=5,10,15$ or 20 , from which we extrapolated to either $j=\infty(x=0)$ or $j=k>j_{0}(x=1 / k)$. Numerous examples which yielded surprisingly high accuracy for a variety of sequences $S_{j}$ in both complete and incomplete cases, attested to the wide applicability of considering $S_{j}$ a smooth function of $1 / j$, even when we were in complete ignorance as to the

Received February 9, 1960; revised April 28, 1960. 
actual analytic expression for $S_{j}=S(1 / j)$ or of a theoretical justification for considering $S(1 / j)$ as an approximate polynomial in $1 / j$.

However, a still further improvement in $m$-point formulas for both complete and partial summation is applicable to a wide class of sequences where $S_{j} \equiv S(1 / j)$ behaves like an even function of $1 / j$. Thus by taking $x^{2}$ as argument instead of $x$, in an $m$-point Lagrangian extrapolation formula for $x=0$ (complete summation) or a value close to 0 (partial summation) based upon those same final $m$ values of $S_{j}$, we should get accuracy equivalent to $(2 m-1)$ th degree instead of $(m-1)$ th degree. As will be seen from the illustrations below, the resulting improvement is of ten quite impressive.

There is no hard and fast classification of all the varied problems to which these newer formulas are applicable. The reason is that even if a problem does not seem offhand to involve a sequence of that even-function type, often with a very slight transformation, regrouping, or alteration, one sees that it really is amenable to this more accurate treatment.

Of course, every sequence to which these improved extrapolation formulas for arguments $1 / j^{2}$ are particularly applicable can also be handled by the earlier formulas employing arguments $1 / j$, because any polynomial in $x^{2}$ is also a polynomial in $x$, but with considerably less accuracy for the same number $m$ of points and the same last $j=j_{0}$. But the converse is not true-we cannot in general expect these newer summation formulas to work well when applied indiscriminately to sequences where the earlier method may give very high accuracy. One way of realizing this is to think of the non-constant part of a well-behaved function of $x$ near $x=0$ being approximated by $C x$. Extrapolation employing $x^{2}=y$ as the variable, near $x=0$, is like extrapolation for $\sqrt{y}$ based upon a polynomial approximation in the variable $y$. But, as anybody who has attempted to interpolate in a table of square roots near zero has found out, $\sqrt{y}$, although continuous at $y=0$, possesses a singularity due to an infinite derivative.

2. Other Related Articles. The idea of the extrapolation to $x=0$ for argument $y=x^{2}$ has been employed for just the linear case in the well-known " $h$-extrapolation process", or "deferred approach to the limit", which has been extensively treated in the literature on the numerical solution of differential equations (first introduced by L. F. Richardson $[3,4])$. The argument $x$ or $h$ corresponds to two conveniently small values of a mesh-length, say $h_{1}$ and $h_{2}$. Richardson's process has been generalized to higher powers beyond $h^{2}$ by several writers, notably G. Blanch, [5] and H. C. Bolton and H. I. Scoins [6]. However, the only reference that was encountered by the writer which was concerned with problems where the approximation might be considered as a purely even function of $h$ having more than a single term, has been M. G. Salvadori [7]. Besides some sets of 2-point coefficients for $h^{2}$ and $h^{4}$-extrapolation, Salvadori tabulates 3-point coefficients for $\left(h^{2}, h^{4}\right)$ - and $\left(h^{4}\right.$, $\left.h^{6}\right)$ - extrapolation, and 4-point coefficients for $\left(h^{2}, h^{4}, h^{6}\right)$ - and $\left(h^{4}, h^{6}, h^{8}\right)$-extrapolation. The values of $h$ are in the form $1 / n_{i}$, where $n_{i}$ are sets of small integers ranging from 2 to 8. Salvadori gives applications to numerical differentiation and integration, as well as to some boundary value problems and characteristic value problems. 
3. Formulas for Complete Summation. In choosing a $j_{0}$ suitable for most complete summation purposes, we wish to obtain a substantial increase in accuracy over the use of the earlier formulas in [1], which has already been proved to be very accurate, without having coefficients that might be too cumbersome. It is also desirable to give exact values rather than decimal values, because in highiy accurate formulas the theoretical or truncation error might be considerably smaller than the computing error arising from the use of rounded decimal entries. But we must also take account of the fact that the fixed points $1 / j^{2}$ in place of the older $1 / j$ makes the exact fractional form of the extrapolation coefficients have around twice as many digits in both numerator and denominator, which adds considerably to the amount of time to do an example.

In the present paper it seems that a very convenient choice is $j_{0}=10$, for all cases ranging from the 2-point through the 7-point. In other words we give formulas for linear through sextic Lagrangian extrapolation formulas for functions of the variable $y=x^{2}$ taken at $x=1 / j$, or arguments $y=1 / j^{2}$ at $j=10,9, \cdots, 10-$ $m+1$ for $m=2(1) 7$. This is equivalent to quadratic through twelfth degree accuracy for even functions in $x=1 / j$. The extrapolation formula to obtain the complete sum $S$ from the partial sums $S_{10}, S_{9}, \cdots, S_{10-m+1}$ is the very simple

$$
S \sim \sum_{i=0}^{m-1} A_{10,10-i}^{(m)} S_{10-i}
$$

The coefficients $A_{10,10-i}^{(m)}$ are given in Table 1 in exact fractional form $B_{10,10-i}^{(m)} / D_{10}^{(m)}$, so that (1) may be most conveniently employed as

$$
S \sim\left(1 / D_{10}^{(m)}\right) \sum_{i=0}^{m-1} B_{10,10-i}^{(m)} S_{10-i}
$$

In no case through $m=7$, does $D_{10}^{(m)}$ have more than ten digits exclusive of final zeros, which is convenient in the division. The values of $A_{10,10-i}^{(m)}$ are given also to 20 decimals in Table 2.

Although the 7-point formulas for $j_{0}=10$ are very accurate, as will be apparent from the examples below, we give also in Table 3 for possible use in some kind of isolated key calculation where extreme accuracy is sought, even at the expense of considerable computing labor, the coefficients in the 11-point formula, ending at $S_{20}$, given exactly, to be employed in

$$
S \sim\left(1 / D_{20}^{(11)}\right) \sum_{i=0}^{10} B_{20,20-i}^{(20)} S_{20-i}
$$

Formula (3) is exact for any even polynomial in $x=1 / j$ up to the 20 th degree. To avoid too much non-essential numerical work, no illustrations were given of the use of Table 3 , since the resulting accuracy is so high by comparison with the results of using Table 1 or 2 , that an excessively large number of significant digits is needed to reveal its full extent. But Table 3 should be kept in reserve for a summation problem requiring unusual precision.

The formula for $A_{j_{0}, j_{0}-i}^{(m)}$ is obtained rather simply from the well-known definition of the $m$-point Lagrangian interpolation coefficients where we have fixed points $1 / j_{0}{ }^{2}, 1 /\left(j_{0}-1\right)^{2}, \cdots, 1 /\left(j_{0}-m+1\right)^{2}$ and set the variable $y=x^{2}=1 / j^{2}$ equal to 0 to correspond to $j=\infty$. 
TABLE 1

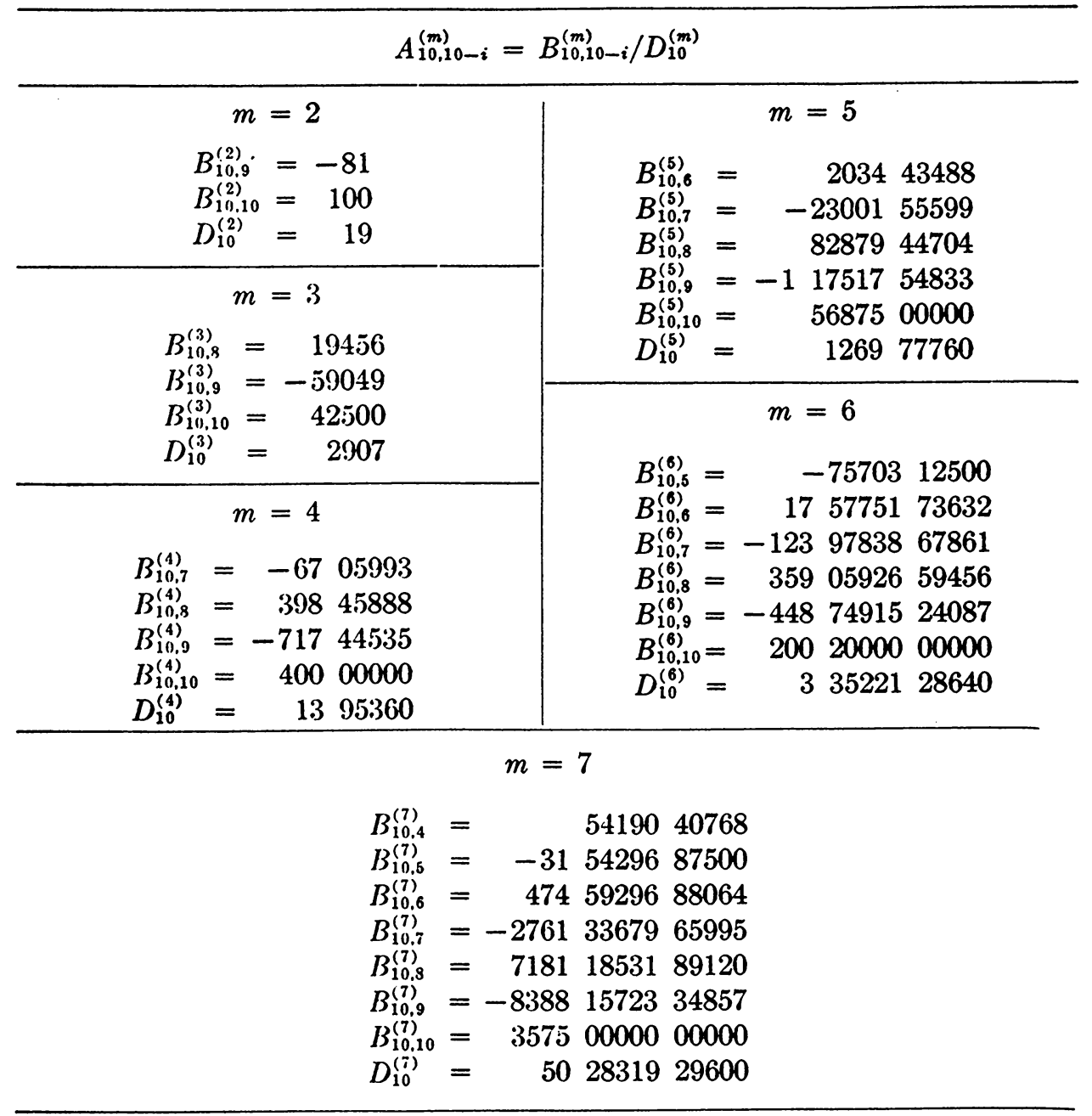

(4)

$$
A_{j_{0}, j c-i}^{(m)}=\frac{(-1)^{m-1}\left(j_{0}-i\right)^{2 m-2}}{\prod_{i=0}^{m-1}\left[\left(j_{0}-k\right)^{2}-\left(j_{0}-i\right)^{2}\right]}
$$

where in $\Pi^{\prime}, k=i$ is omitted.

\section{Illustrations of Complete Summation.}

A. Example 1. Considering the circle as the limiting case of inscribed regular polygons of $j$ sides, as $j \rightarrow \infty$, the quantity $\pi$ is the limit of the semi-perimeter, $j \sin \alpha$, where $\alpha=180^{\circ} / j=\pi / j$, as $j \rightarrow \infty$. Now the approximation $S_{j}=j \sin \alpha=$

* Although this example affords a splendid illustration of the improvement of $\left(1 / j^{2}\right)$ extrapolation over $(1 / j)$-extrapolation, it suffers from the aesthetic defect of having the value of $\pi$ occurring implicitly in every $S_{j}$ in the various powers of $\alpha$ needed to compute $\sin \alpha$. In other words, there is definitely something "circular" in this example. 
TABLE 2

\begin{tabular}{|c|c|c|c|c|}
\hline \multicolumn{5}{|c|}{$A_{10,10-i}^{(m)}$ in Decimal Form } \\
\hline & $m=2$ & & \multirow{3}{*}{$\begin{array}{l}A_{10.5}^{(6)} \\
A_{10,6}^{(6)} \\
A_{10,7}^{(6)} \\
A_{10,8}^{(6)} \\
A_{10,9}^{(6)} \\
A_{10,10}^{(6)}\end{array}$} & \multirow{3}{*}{$\begin{array}{rrr}= & -0.22583030395530395530 \\
= & 5.2435564435 \text { 56443 } 55644 \\
= & -36.9840436201189877660 \\
= & 107.11111749543122092142 \\
= & -133.86654446318000884564 \\
= & 59.7217 \pm 444826663608913\end{array}$} \\
\hline $\begin{array}{l}A_{10,9}^{(2)} \\
A_{10,10}^{(2)}\end{array}$ & $\begin{array}{l}=-4.2631578947 \\
=\quad 5.2631578947\end{array}$ & $\begin{array}{ll}368+2 & 10526 \\
36842 & 10526 \\
\end{array}$ & & \\
\hline $\begin{array}{l}A_{10,8}^{(3)} \\
A_{10,9}^{(3)} \\
A_{10,10}^{(3)}\end{array}$ & $\begin{aligned} & m=3 \\
= & 6.6928104575 \\
= & -20.3126934984 \\
= & 14.6198830409\end{aligned}$ & 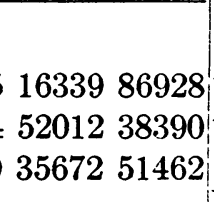 & & \\
\hline \multicolumn{3}{|c|}{$m=4$} & \multirow{3}{*}{$\begin{array}{l}A_{10,4}^{(7)} \\
A_{10,5}^{(7)} \\
A_{10,6}^{(7)} \\
A_{10,7}^{(7)} \\
A_{10,8}^{(7)} \\
A_{10,9}^{(7)} \\
A_{10,10}^{(7)}\end{array}$} & $m=7$ \\
\hline $\begin{array}{l}A_{10,7}^{(4)} \\
A_{10.8}^{(4)} \\
A_{10,9}^{(4)} \\
A_{10,10}^{(4)}\end{array}$ & $\begin{array}{lr}= & -4.8059232026 \\
= & 28.5559912854 \\
= & -51.4165054179 \\
= & 28.6664373351\end{array}$ & $\begin{array}{ll}14379 & 08497 \\
03050 & 10893 \\
56656 & 34675 \\
67985 & 32278\end{array}$ & & $\begin{array}{lr}= & 0.01077704188815299926 \\
= & -0.62730639987581432029 \\
= & 9.43840159840159840160 \\
= & -54.91570113290395153140\end{array}$ \\
\hline $\begin{array}{l}A_{10,6}^{(5)} \\
A_{10,7}^{(5)} \\
A_{10,8}^{(5)} \\
A_{10,9}^{(5)} \\
A_{10,10}^{(5)}\end{array}$ & $\begin{array}{rr} & m=5 \\
= & 1.6021978021 \\
= & -18.1146336098 \\
= & 65.2708372237 \\
= & -92.5497097523 \\
= & 44.7913083361\end{array}$ & $\begin{array}{ll}97802 & 19780 \\
54198 & 08949 \\
78400 & 24899 \\
21981 & 42415 \\
99977 & 06685\end{array}$ & & $\begin{array}{lr}= & 142.81482332724162789522 \\
= & -166.81830925 \pm 1 \quad 1662640765 \\
= & 71.09731481936 .504296325\end{array}$ \\
\hline
\end{tabular}

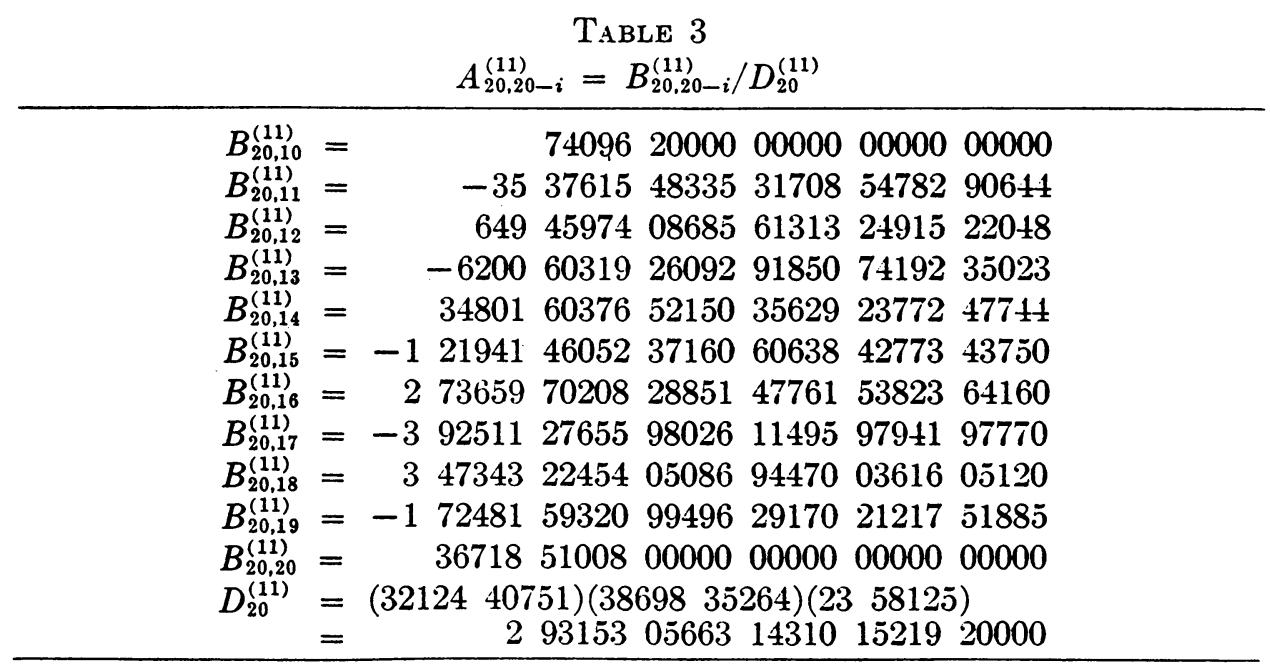

$S(1 / j)$ is seen to be an even function of $1 / j$ which equals $\pi$ for $1 / j=0$. Therefore we expect an $m$-point Lagrange polynomial approximation for variable $1 / j^{2}$ to be considerably more accurate than a polynomial in $1 / j$. Following are the values of the semi-perimeters $j \sin \alpha$ to $25 \mathrm{D}$, which were obtained from a table of $\sin \alpha$ to $30 \mathrm{D}$ originally published by Herrmann [8]. For $j=4(1) 6,9,10, \sin \alpha$ was copied 
from Herrmann's table, and for $j=7,8, \sin \alpha$ was computed by Taylor's theorem employing Herrmann's entries as key values:

\begin{tabular}{r|rrrrr}
\hline$j$ & \multicolumn{4}{|c}{$s_{j}:$ Semi-perimeter $=j \sin \alpha$} \\
\cline { 2 - 6 } 4 & 2.82842 & 71247 & 46190 & 09760 & 33774 \\
5 & 2.93892 & 62614 & 62365 & 64584 & 35298 \\
6 & 3.00000 & 00000 & 00000 & 00000 & 00000 \\
7 & 3.03718 & 61738 & 22906 & 84333 & 03783 \\
8 & 3.06146 & 74.589 & 20718 & 17382 & 76799 \\
9 & 3.07818 & 12899 & 31018 & 59739 & 68965 \\
10 & 3.09016 & 99437 & 49474 & 24102 & 29342 \\
\hline
\end{tabular}

In the above values of $S_{j}$, as well as $S_{j}$ given in the other examples, the accuracy of the last few places, although highly probable, is still not absolutely guaranteed. However, in every example the values of $S_{j}$ are certainly correct up to the number of places needed to guarantee that the "computational error" in the final answer (which is due to the error in the $S_{j}$ multiplied by the extrapolation coefficients $\left.A_{10, j}^{(m)}\right)$ is appreciably less than the deviation of the answer from the true value. This latter "truncuting error" is thus made to stand out clearly, and it indicates the theoretical accuracy of the extrapolation formula, regardless of the number of places carried in the work. In practice we do not often know at the outset of an example just how many placess are needed in the $S_{j}$ to assure us that the computing error will be dominated by the truncating error. Sometimes when the theoretical accuracy turns out to be unexpectedly fine, the example must be done again, carrying more places, to prevent the computing error from obscuring the truncating error.

The results of the extrapolations employing (1) or (2), for $m=7$, gave for $\pi$, (whose true value to $20 \mathrm{D}$ is 3.14159265358979323846 ), the answer 3.14159 $26533589793179 \ldots$ which is correct to within a unit in the 16 th decimal. The extent of the improvement over the earlier $(1 / j)$-extrapolation formulas is apparent from the result of $3.1+15$ ) $280 \ldots$ obtained by the corresponding 7 -point $(1 / j)$ extrapolation cocflicients, which deriates from $\pi$ by $1 \frac{1}{2}$ units in the 7 th decimal. In other words, the error in the use of this newer formula is only around $0.4 \cdot 10^{-9}$ of that in the older one. The greater power of this newer method in this present example may be further illustrated even for $m=4$, where $\left(1 / j^{2}\right)$-extrapolation yields $3.141592650 \ldots$, or accuracy to around $\frac{1}{3}$ of a unit in the 8 th decimal, whereas the corresponding $t$-point $(1 / j)$-extrapolation formula gives no better than 3.1411 $\ldots$, which is off by $\frac{1}{2}$ of a unit in the 3rd decimal. In fact, the answer even by 2-point $\left(1 / j^{2}\right)$-extrapolation, namely $3.1413 \ldots$, is still better than the above 3.1411... .

It is interesting to note that the use of $\left(1 / j^{2}\right)$-extrapolation on the semi-perimeters gives this great improvement only for the inscribed polygons, and it will not work well for the circumscribed polygons, upon which it was also tried. A reason that would lead us to expect poor extrapolation results, even though the corresponding semi-perimeter $j \tan \alpha$ is still an even function of $1 / j$, is that the series for $\tan \alpha$ converges poorly by comparison with $\sin \alpha$. Thus for $\alpha=\pi / 4$, occurring in $S_{j}=S_{4}$, the remainder after the term involving the sixth power of $1 / j^{2}$, is con- 
siderably greater for $j \tan \alpha$, so that the use of (1) or (2) for $m=7$ is not nearly so good as for $j \sin \alpha$.

B. Example 2. The sequence for Euler's constant

$$
\gamma=\lim _{j \rightarrow \infty}\left\{\sum_{r=1}^{j}(1 / r)-\log j\right\}=0.57721566490153286061 \text { to } 20 \mathrm{D}
$$

has been treated earlier by $(1 / j)$-extrapolation ([1], p. 358). Applying (1) or (2), for seven points, directly to $S_{j}=\sum_{r=1}^{j}(1 / r)-\log j$ yields the very inaccurate 0.593 , the reason being that $S_{j}$ does not behave like an even function of $1 / j$. The older $(1 / j)$-extrapolation formulas, employing $j_{0}=10$, gave $0.5772141 \ldots$ and $0.5772156695 \ldots$ by the 4 - and 7-point formulas with respective errors of around $1 \frac{1}{2} \cdot 10^{-6}$ and $\frac{1}{2} \cdot 10^{-8}$. To improve upon these results we must modify our $S_{j}$ sequence into an even function of $1 / j$ having the same limit $\gamma$. This is easily accomplished by replacing the last $1 / r$ in the summation, namely $1 / r=1 / j$, by half its value, or $1 / 2 j$. At first sight there is an apparent motivation in that the new finite summation is suggestive (at one end anyhow) of the more accurate trapezoidal rather than rectangular approximation to the integral $\int_{1}^{j}(1 / r) d r$. This trapezoidal motivation happens to lead to the correct choice in this present example, but in general it does not yield a sequence that is even in $(1 / j)$. The true motivation lies in the Euler-Maclaurin summation formula applied to $\log j$. The general formula is expressible as

$$
\begin{aligned}
\frac{1}{w} \int_{a}^{a+j w} f(x) d x=\left(\frac{1}{2} f_{0}\right. & \left.+f_{1}+f_{2}+\cdots+f_{j-1}+\frac{1}{2} f_{j}\right)-\frac{w}{12}\left(f_{j}^{\prime}-f_{0}{ }^{\prime}\right) \\
& +\frac{w^{3}}{720}\left(f_{j}^{\prime \prime \prime}-f_{0}^{\prime \prime \prime}\right)-\frac{w^{5}}{30240}\left(f_{j}{ }^{v}-f_{0}{ }^{v}\right)+\cdots[9] .
\end{aligned}
$$

Now (5) does not denote a complete equality, since the Euler-Maclaurin formula is an asymptotic expression that is given with a remainder term. Employing (5) heuristically for $w=1, a=1$ and $f(x)=1 / x$, the right member of (j), exclusive of the $\left(\frac{1}{2} f_{0}+f_{1}+\cdots+f_{j-1}+\frac{1}{2} f_{j}\right)$ and an undisclosed remainder term, is an even function of $1 /(j+1)$, from which, replacing $j$ by $j-1$,

$$
\int_{1}^{j}(1 / x) d x-\left(\frac{1}{2}+\frac{1}{2}+\frac{1}{3}+\cdots+\frac{1}{j-1}+\frac{1}{2 j}\right)
$$

is an even function of $1 / j$, so that the same is true of the sequence

$$
\mathrm{S}^{\prime} \equiv\left(1+\frac{1}{2}+\frac{1}{3}+\cdots+\frac{1}{j-1}+\frac{1}{2 j}\right)-\log j
$$

whose limit, as $j \rightarrow \infty$, is also equal to $\gamma .^{*}$

Since the older $m$-point $(1 / j)$-extrapolation formula is linear in $S_{j}\left(\right.$ or $S_{j}{ }^{\prime}$ ) and

* The reader is cautioned that the above heuristic demonstration is not to be understood as a proof that we have a convergent infinite series in $\left(1 / j^{2}\right)$ from which we can "prove" that the "constant" term in $S_{j}^{\prime}$ is $\gamma$ by taking the limit as $j \rightarrow \infty$. The fallacy there would be in that there is no "constant" term because the $f_{0}, f_{0}^{\prime}, f_{0}^{\prime \prime}$ ", $\cdots$ terms in (5) yield for $f(x)=1 / x$ a divergent sequence. Actually $S_{i}$ ' is defined only up to any fixed order derivative, say $f_{j-1}^{(p)}$, and it then consists of terms in $1 / j^{2}$, constant terms and an integral formula for the remainder. 
yields exactly zero for any polynomial in $1 / j$ having no constant term, up to the $(m-1)$ th degree, the above-mentioned 4- and 7 -point results will not be changed by use of $S_{j}^{\prime}$ instead of $S_{j}$. But the improvement is very noticeable when $S_{j}^{\prime}$ is employed with $\left(1 / j^{2}\right)$-extrapolation. Following are the terms in the modified sequence $S_{j}{ }^{\prime}$ to $20 \mathrm{D}$ :

\begin{tabular}{|c|c|}
\hline$j$ & $S_{j^{\prime}}=\sum_{r=1}^{j-1} \frac{1}{r}+\frac{1}{2 j}-\log j$ \\
\hline 4 & $0.5720389722 \quad 13442 \quad 71450$ \\
\hline 5 & $0.57389 \quad j 42089923295873$ \\
\hline 6 & 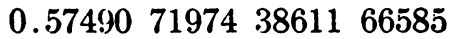 \\
\hline 7 & $\begin{array}{lllll}0.575 .51 & 84223 & 73258 & 12347\end{array}$ \\
\hline 8 & 0.57.991 56011 7730692889 \\
\hline 9) & $\begin{array}{llll}0.57618 & 81210 & 76479 & 02991\end{array}$ \\
\hline 10 & $\begin{array}{lllll}0.576 .38 & 31609 & 74208 & 28424\end{array}$ \\
\hline
\end{tabular}

The use of the 7 -point formula in (1) or (2), where $j_{0}=10$, upon $S_{j}{ }^{\prime}$, gave an answer of 0.57721 56itity $0143 \ldots$ which is correct to a unit in the 13th decimal (i.e., i) placess more than $(1 / j)$-extrapolation). Use of just the 4-point formula in (1) or (2) galve all answer as good as $0.57721566475 \ldots$ which is correct to within 1.12 units in the 10 th decimal (i.e., 4 places more than $(1 / j)$-extrapolation).

(:. Lirumple 3. 1 different type of secfuence is encountered in the evaluation of the definite integral $\int_{0}^{1} \frac{1}{1+x} d x=\log 2$, whose value to $20 \mathrm{D}$ is 0.6931471805 5)(9).5 30942. One obvious se(puence to consider is $S_{j}$ which is formed by dividing the interval $(0,1)$ into $j$ equally spaced intervals and letting $S_{j}$ be the sum of the rectangles of height $1 /[1+(r-1) / . j]$ and width $1 / j$, for $r=1(1) j$, but that fails to behave as an even function of $1 / j$. However, the trapezoidal rule, or

$$
S_{j}{ }^{\prime}=\frac{1}{j}\left(\frac{1}{2}+\frac{1}{1+1 / j}+\frac{1}{1+2 / j}+\cdots+\frac{1}{1+(j-1) / j}+\frac{1}{4}\right),
$$

arcording to the buler-Maclaurin formula (5), where now $w=1 / j, a=0$, and both $f_{j}{ }^{(p)}$ is fixed as well as $f_{0}{ }^{(p)}$, being at the endpoints 1 and 0 , is seen to have a truncating crror that is formally a series in $1 / j^{2}$. The values of $S_{j}{ }^{\prime}$, in either exact form, or to 201), are als follows:

\begin{tabular}{|c|c|}
\hline j & $S_{j^{\prime}}=\frac{1}{i}\left(\sum_{i}^{1}+\sum_{r=1}^{j-1} \frac{1}{1+r / j}+\frac{1}{i}\right)$ \\
\hline$t$ & $11711680=0.69702380952380952381$ \\
\hline j & $1752 \div 20=0.69563+92063492063492$ \\
\hline 6 & 9631 1:3S60 $=0.69+87734487734487734$ \\
\hline 7 & $250241 / 360: 360=0.694419469+1946941947$ \\
\hline 8 & $200107: 2$ SS $28 S=0.69412 \quad 185037185037185$ \\
\hline 9 & $566803: 816816=0.69391760200583729995$ \\
\hline 10 & $16150+821 / 232792560=0.693771403175+2794323$ \\
\hline
\end{tabular}

The 4 - and 7 -point $(1 / j)$-extrapolation, $j_{0}=10$, gave values of $0.6931486 \ldots$ and $0.693147176 \ldots$, correct to $1 \frac{1}{2}$ units in the 6 th decimal and $\frac{1}{2}$ unit in the 8 th 
decimal respectively. The $\left(1 / j^{2}\right)$-extrapolation was performed for every $m$-point formula from $m=2$ through $m=7$, with the following results:

\begin{tabular}{|c|c|c|c|c|c|}
\hline$m$ & ralue of $S$ & decialion & $m$ & ralue of $S$ & deriation \\
\hline $\begin{array}{l}2 \\
3 \\
4\end{array}$ & $\begin{array}{llll}0.69314 & 81 \ldots & \\
0.69314 & 7188 \ldots & \\
0.69314 & 71807 & 1 \ldots\end{array}$ & $\begin{array}{c}10^{-6} \\
10^{-8} \\
1 \frac{1}{2} \cdot 10^{-10}\end{array}$ & $\begin{array}{l}5 \\
6 \\
7\end{array}$ & 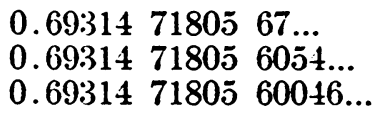 & $\begin{array}{l}10^{-11} \\
3 \cdot 10^{-12} \\
10^{-13}\end{array}$ \\
\hline
\end{tabular}

The improvement over $(1 / j)$-extrapolation in the 4 - and 7 -point results is by four and five places respectively.

D. Example 4. A somewhat more sophisticated application of $\left(1 / j^{2}\right)$-extrapolation is in the summation of the series for Catalan's constant, or

$$
T_{2}=1-\frac{1}{3^{2}}+\frac{1}{5^{2}}-\frac{1}{7^{2}}+\cdots
$$

H. T. Davis [10] gives a full discussion of Catalan's constant, including an account of the earlier work of J. W. L. Glaisher, and he also reprints Glaisher's 32-decimal value of $T_{2}=0.9159655941772190150 \tilde{J} 4603 \tilde{j} 1493238$. Since the series for $T_{2}$ is absolutely convergent, it may be regrouped as

$T_{2}=1+\left(-\frac{1}{3^{2}}+\frac{1}{\overline{3}^{2}}\right)+\left(-\frac{1}{7^{2}}+\frac{1}{9^{2}}\right)+\cdots+\left(-\frac{1}{(4 r-1)^{2}}+\frac{1}{(4 r+1)^{2}}\right)+\cdots$

The general term $u_{r}, r>0$, of $T_{2}$ is equal to $\frac{-16 r}{\left(16 r^{2}-1\right)^{2}}$, which is an odd function of $r$ or $1 / r$. Thus, as in the preceding example, employing ( 0 ) with $w=a=1$, the modified sum

$$
S_{j}^{\prime}=S_{j}-\frac{1}{2} u_{j}=1-\sum_{r=1}^{j-1} \frac{16 r}{\left(16 r^{2}-1\right)^{2}}-\frac{1}{2}\left(\frac{16 j}{\left(16 j^{2}-1\right)^{2}}\right)
$$

is again seen to be formally an even function of $1 / j$, having the same limit $S$ which is approached by $S_{j}{ }^{*}$ The values of $S_{j}{ }^{\prime}$ to $20 \mathrm{D}$ are as follows:

\begin{tabular}{c|cc}
\hline & $s_{j^{\prime}}=1-\sum_{r=1}^{j-1} \frac{16 r}{\left(16 r^{2}-1\right)^{2}}-\frac{1}{2}\left(\frac{16 j}{\left(16 j^{2}-1\right)^{2}}\right)$ \\
\hline 4 & 0.91798698317333085103 \\
5 & 0.91724361005416302747 \\
6 & 0.91684717576686806945 \\
7 & 0.91661065547755203321 \\
8 & 0.91645816017196679489 \\
9 & 0.91635407246123031205 \\
10 & 0.91627985019173237910 \\
\hline
\end{tabular}

* Although in Example 4 we know the explicit formula for $\int_{1}^{j} f(x) d x$, we may expect this principle to be applicable also in cases where $\int_{1}^{j} f(x) d x, f(x)$ odd, or for that matter also $f_{j}{ }^{(p)}$ for odd $p$, is not known in closed form, and where $S_{j}$ may still be regarded formally as a series in $1 / j^{2}$. 
Use of the 7-point $(1 / j)$-extrapolation, $j_{0}=10$, upon either $S_{j}$ or $S_{j}{ }^{\prime}$, while not identical in accuracy, because now the difference of $\frac{1}{2}\left(16 j /\left(16 j^{2}-1\right)^{2}\right)$ is no longer an exact polynomial in $1 / j$, gave results very close to each other, namely 0.91596 $55973 \ldots$ and $0.9159655980 \ldots$ with respective deviations of $\frac{1}{3} \cdot 10^{-8}$ and $\frac{2}{5} \cdot 10^{-8}$. The use of $\left(1 / j^{2}\right)$-extrapolation, i.e., (1) or (2), for $m=7$, while giving the poorer answer of $0.9159674 \ldots$ with a deviation of $2 \cdot 10^{-6}$ in working with the $S_{j}$ sequence (as was to be expected), gave upon working with the $S_{j}{ }^{\prime}$ sequence the highly accurate $0.91596559417714 \ldots$, which is correct to $\frac{4}{5} \cdot 10^{-13}$, showing a gain in accuracy of around 5 places.

5. Formulas for Partial Summation. Given the first ten terms of a sequence $S_{j}$ which behaves as an even function of $1 / j$, we might wish to find by $\left(1 / j^{2}\right)$-extrapolation $S_{n}, n>10$, instead of going to the limit as $j \rightarrow \infty$. The purpose of this section is to improve what was accomplished in [2] where just $(1 / j)$-extrapolation was employed. The $m$-point formula for $S_{n}$ which occurs usually as a sum of the form $\sum_{r=0}^{n} u_{r}$, is obtained by setting $x=1 / n^{2}$ in the Lagrange interpolation coefficients whose fixed points are $1 / j_{0}{ }^{2}, 1 /\left(j_{0}-1\right)^{2}, \cdots, 1 /\left(j_{0}-m+1\right)^{2}$. In the present instance, in order to avoid too much tabulation, since now besides $j_{0}$ and $m, n$ is also a variable, being no longer just $\infty$, we consider a choice of $j_{0}$ and $m$ which shall be suitable for most problems and which shall give a substantial increase in accuracy over the $(1 / j)$-extrapolation formulas previously given which were based upon $j_{0}=10$ and $m=7$ [2]. Thus it is natural to take $j_{0}=10$ and $m=7$ for these present formulas also. The argument $n=11(1) 25(5) 100,200,500$, 1000 , and all coefficients are given exactly. This range of $n$ is not quite so extensive as in the previous paper because the arguments $1 / j^{2}$ in place of $1 / j, j=4,5, \cdots$, $10, n$, increase the labor in computing the exact forms, which also have considerably greater bulk in figures. To find $S_{n} \equiv S(n)$, we employ the extrapolation formula in the following form:

$$
S(n)=\sum_{j=4}^{10} A_{j}(n) S_{j}
$$

Every set of cocflicients $A_{j}(n)$ is given in the exact fractional form of $C_{j}(n) / D(n)$ where $D(n)$ is the least common denominator for each $n$. Thus it may help the computer to have

$$
S(n)=(1 / D(n)) \sum_{j=4}^{10} C_{j}(n) S_{j} .
$$

In $(\boldsymbol{b})$ and $(\bar{T})$ the $j_{0}=10$ is understood as well as $m=7$. When also $n$ is understood, we may employ for $(7)$ the somewhat more concise

$$
S_{n}=(1 / D) \sum_{j=4}^{10} C_{j} S_{j}
$$

In $(7)$, or $\left(7^{\prime}\right)$, the $D(n)$, or $D$, is given also in the form of factors having no more than 10 digits, exclusive of terminal 0 's, to facilitate the divisions on a ten-bank desk calculator. The $C_{j}(n)$ and $D(n)$ are shown in Table 4. 


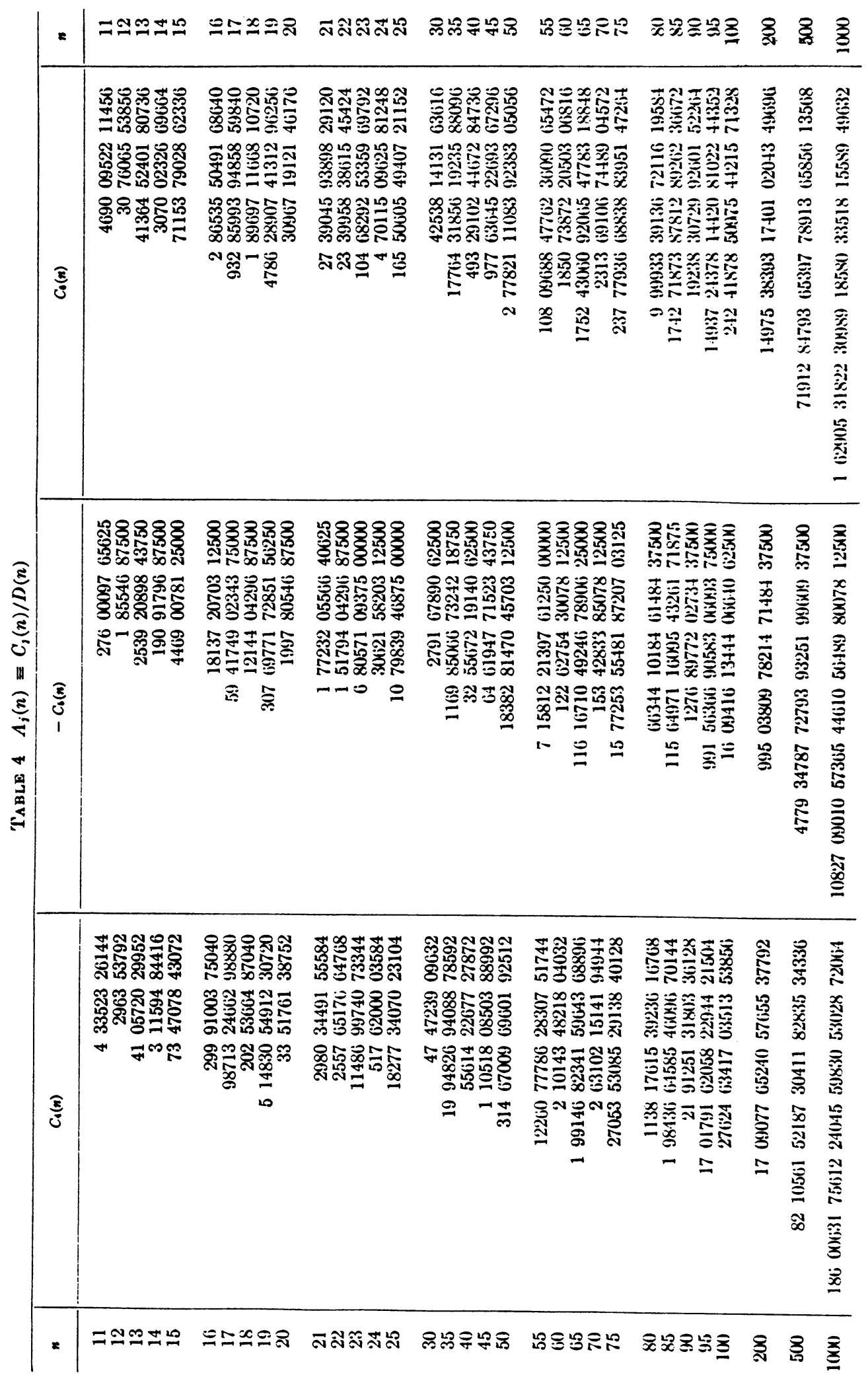




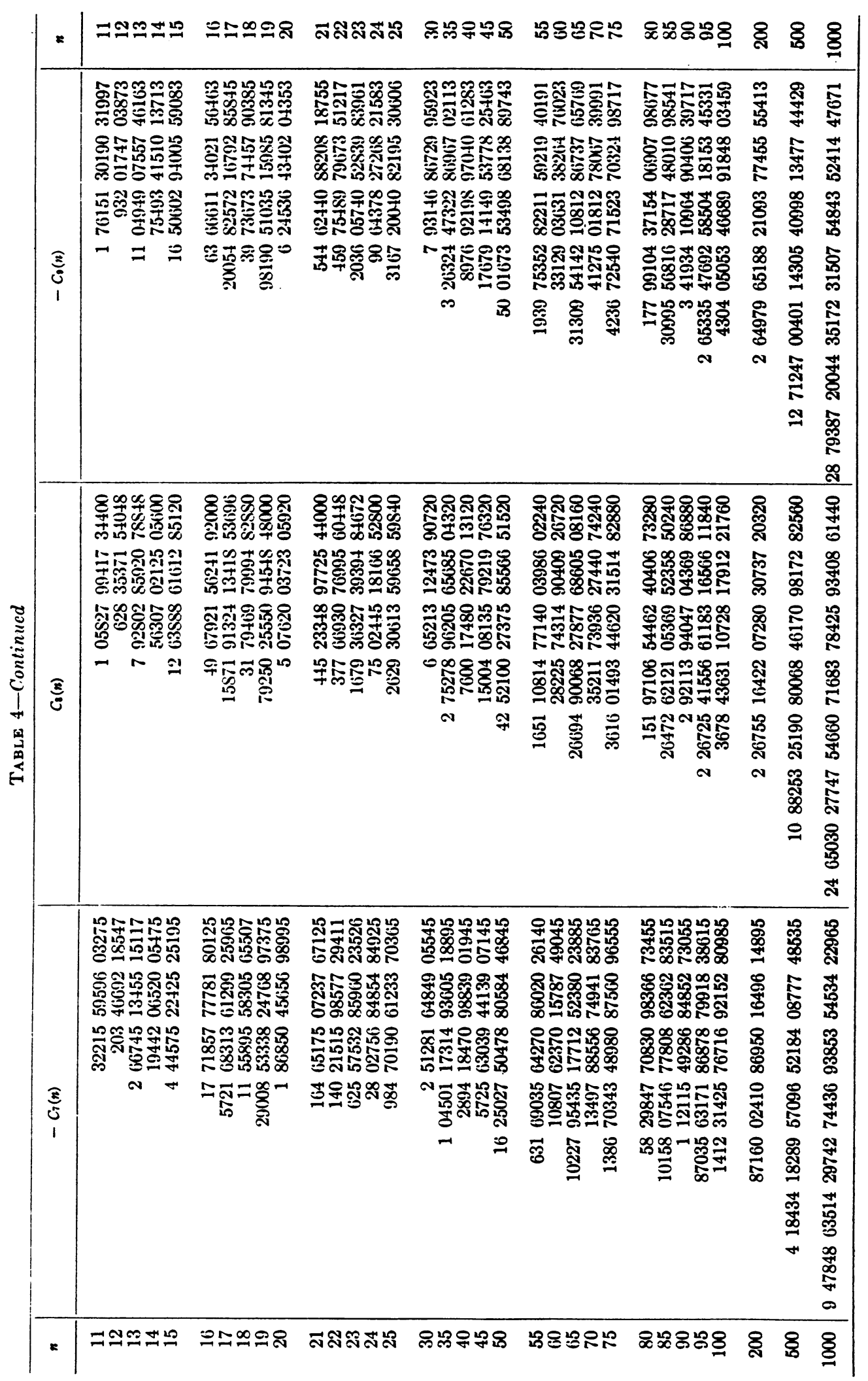




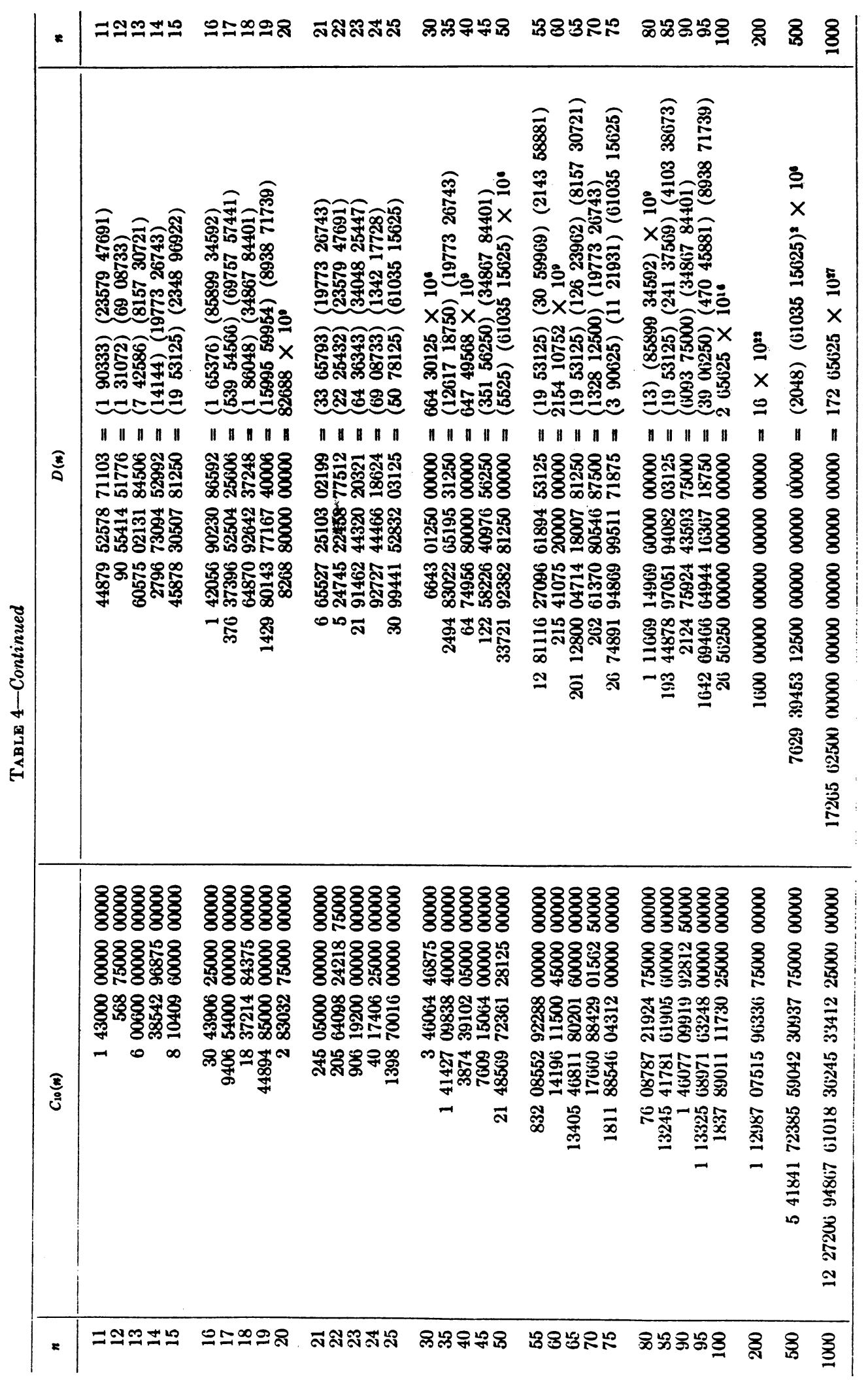


The coefficients $A_{j}(n)=C_{j}(n) / D(n)$ were calculated directly from the formula

$$
A_{j}(n)=\frac{j^{12}}{n^{12}} \frac{\prod_{k=4}^{10}\left(n^{2}-k^{2}\right)}{\prod_{k=4}^{10}\left(j^{2}-k^{2}\right)},
$$

where $k=j$ is absent from $\Pi^{\prime}$. Both the calculation of $A_{j}(n)$ and the determination of $D(n)$ was facilitated by expressing each of the factors in the right member of $(8)$ in terms of powers of primes.

To facilitate the use of (8) for desired values of $n$ other than in this present table, we notice that we may express $A_{j}(n)$ as

$$
\begin{aligned}
A_{j}(n) & =B_{j} \cdot \frac{\prod_{k=4}^{10}\left(n^{2}-k^{2}\right)}{n^{12}}, \text { where } \\
B_{j} & =\frac{j^{12}}{\prod_{k=4}^{10}\left(j^{2}-k^{2}\right)}
\end{aligned}
$$

is independent of $n$. The exact, as well as 30 decimal, values of the fundamental quantities $B_{j}$ are given in the following Schedule 1.

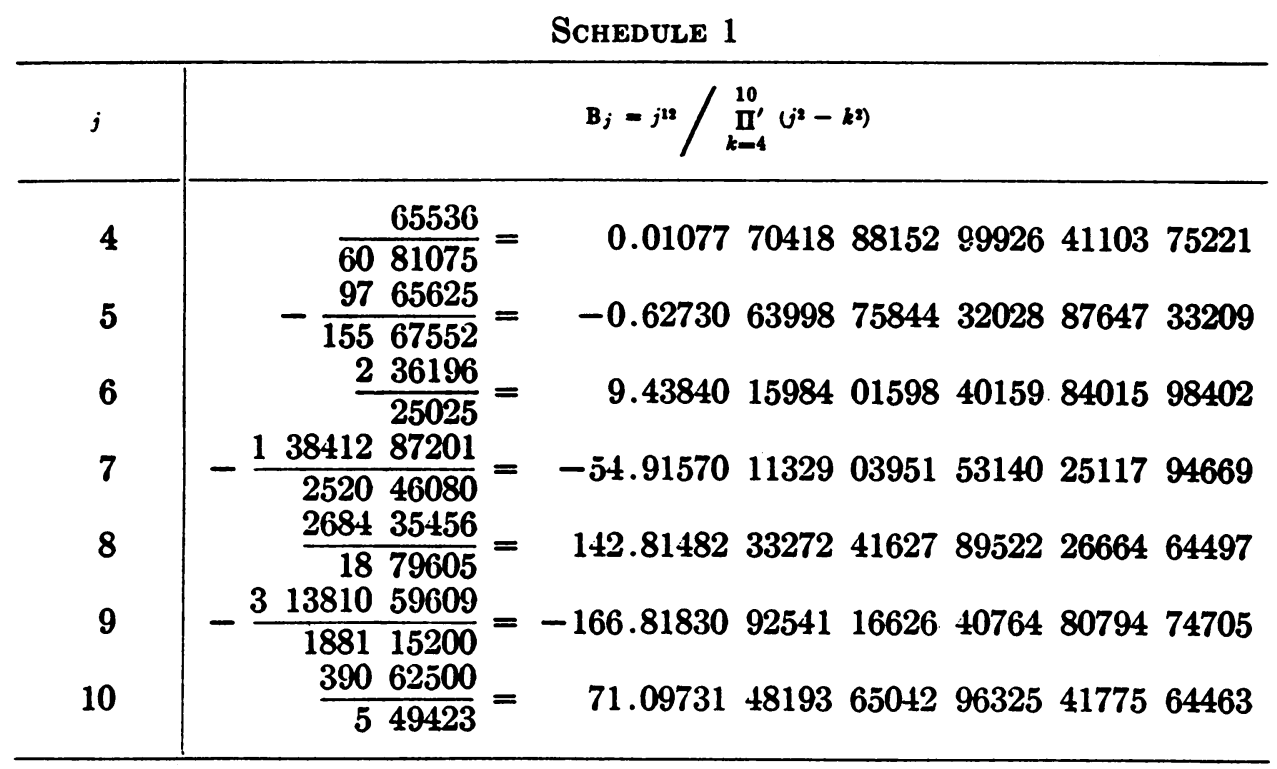

6. Illustrations of Partial Summation.

A. Example 5. Suppose that in Example 1 above, instead of passing to the limit as $j \rightarrow \infty$ to obtain $\pi$, we wished to calculate $S_{20}$, or the semi-perimeter of a 20 sided regular polygon from the semi-perimeters of the 4 - through 10-sided regular polygons. We have $S_{20}=20 \sin 9^{\circ}$, whose value to $20 \mathrm{D}$ is 3.128689300804617 
38020. Using the same values of $S_{j}$ as in Example 1, we find by the earlier method of $(1 / j)$-extrapolation [2] $S_{20}=3.1286893076 \ldots$ which is correct to around a unit in the 8 th decimal. But use of the present tables for $\left(1 / j^{2}\right)$-extrapolation in (6) or $(7)$, for $n=20$, yields the highly accurate $S_{20}=3.128689300804617359 \ldots$, correct to about 2 units in the 17th decimal, showing a gain of around 9 places.

B. Example 6. As an illustration of a different type of problem that does not correspond to one in complete summation, consider the case where from the first few known zeros of some higher mathematical function, we wish to obtain the value of some later zero, say the $n$ th. As will be seen below, there are circumstances when it is preferable to choose as the sequence $S_{j}, j \leqq j_{0}$, from which to extrapolate, some suitable even function of $1 / j$ which may not be a function of the $j$ th root, and yet from $S_{j}, j>j_{0}$, the $j$ th root, is readily obtainable.

Consider the problem of finding the later zeros of the spherical Bessel functions $J_{2 m+1}(z)$ from either tabulated earlier zeros or some other suitable function of $m$. In the general asymptotic formula for $z_{\nu}^{(n)}$, the $n$th zero of $J_{\nu}(z) \cos \alpha-Y_{\nu}(z) \sin \alpha$, namely,

$$
\begin{aligned}
z_{\nu}^{(n)}=\left(n+\frac{1}{2} \nu-\frac{1}{4}\right) \pi-\alpha- & \frac{4 \nu^{2}-1}{8\left\{\left(n+\frac{1}{2} \nu-\frac{1}{4}\right) \pi-\alpha\right\}} \\
& -\frac{\left(4 \nu^{2}-1\right)\left(28 \nu^{2}-31\right)}{384\left\{\left(n+\frac{1}{2} \nu-\frac{1}{4}\right) \pi-\alpha\right\}^{3}}-\cdots[11],
\end{aligned}
$$

set $\alpha=0$ and $\nu=2 m+\frac{1}{2}$. Then from (11) it is apparent that

$$
S_{n+m} \equiv(n+m)\left[z_{2 m+1}^{(n)}-(n+m) \pi\right]
$$

has a formal expansion in even powers of $1 /(n+m)$, which could serve as the basis of an extrapolation formula.

However, after searching for ready-made tables of $z_{2 m+1}^{(n)}$, none were found capable of testing the full potentialities of Table 4. To avoid extra labor, we shall first illustrate this principle of $\left(1 / \nu^{2}\right)$-extrapolation with a smaller example limited to the available published $6 \mathrm{D}$ values of $z_{9 / 2}^{(n)}$ as far as $n=6$ [12]. The problem is to calculate $z_{9 / 2}^{(n)}$ for $n=6$, whose published value is 24.727566 , from the four preceding values of $z_{9 / 2}^{(2)}=11.704907, z_{9 / 2}^{(3)}=15.039665, z_{9 / 2}^{(4)}=18.301256$ and $z_{9 / 2}^{(5)}=$ 21.525418. In other words, since $m=2$, the problem is to find $S_{3}$ from $S_{4}, S_{5}, S_{6}$ and $S_{7}$, from which $z_{9 / 2}^{(6)}$ is found from (12). From (8), with $\prod_{k=4}^{10}$ replaced by $\prod^{\prime 7}{ }_{k=4}^{7}$, we find $A_{4}(8)=-\frac{97}{2112}, A_{5}(8)=\frac{546875}{1081344}, A_{6}(8)=-\frac{19683}{11264}$ and $A_{i}(8)=$ $\frac{823543}{360448}$ from which $S_{8}=\sum_{j=4}^{7} A_{j}(8) S_{j}=-3.241393$. Finally, from (12), $z_{9 / 2}^{(6)}$ is found to be 24.727567 , which deviates by only $10^{-6}$ from the published value.* Comparing with $(1 / \nu)$-extrapolation based upon those same values of $S_{4}-S_{i}$, and where $A_{4}(8)=-\frac{1}{8}, A_{5}(8)=\frac{12}{12} \frac{5}{8}, A_{6}(8)=-\frac{81}{32}, A_{7}(8)=\frac{34}{12} \frac{3}{8}$, we find $S_{8}=$ -3.241225 , from which $z_{9 / 2}^{(6)}$ is found to be $24.727 j 88$, which deviates by 0.000022 from the published value.

* Since we started with $6 \mathrm{D}$ values, it is not possible to estimate from this example the possibly higher theoretical accuracy in $\left(1 / \nu^{2}\right)$-extrapolation, which is just the truncation error when the example is done with a sufficiently large number of places both initially and in the course of the work. 
For a similar example employing Table 4, and revealing the full accuracy of (6) or $(7)$, we choose a modification of $S_{n+m}$, say $\bar{S}_{n+m}$, where

$$
\bar{S}_{n+m}=(n+m)\left[\bar{z}_{2 m+1}^{(n)}-(n+m) \pi\right]
$$

and where now $\bar{z}_{2 m+\frac{1}{3}}^{(n)}$, instead of being the $n$th zero of $J_{2 m+\frac{1}{1}}(x)$, is defined as a preassigned number of terms of the right member of (11) (for $\alpha=0, \nu=2 m+\frac{1}{2}$ ) which is the same for every $n$. For the lowest values of $n$, there will be considerable deviation between the true value of the root $z_{2 m+1}^{(n)}$ and the function $\bar{z}_{2 m+1}^{(n)}$ which is $(n+m) \pi+$ an exact odd polynomial in $1 /(n+m)$, making $\bar{S}_{n+m}$ an exact even polynomial in $1 /(n+m)$. But at the inconvenience of having to compute $\bar{S}_{n+m}$ for the initial values of $n$, we may employ (6) or (7) to extrapolate for $\bar{S}_{n+m}$ for some larger $n$ to get $\bar{z}_{2 m+1}^{(n)}$ which will agree with the true value of the root $z_{2 m+1}^{(n)}$ to very high accuracy. Taking (11) as far out as $1 /\left\{\left(n+\frac{1}{2} \nu-\frac{1}{4}\right) \pi-\alpha\right\}^{9}$, we have for $\alpha=0, \nu=2 m+\frac{1}{2}$ and $\mu \equiv 4 \nu^{2}=(4 m+1)^{2}$,

$$
\begin{aligned}
\bar{S}_{n+m}= & -\frac{\mu-1}{2^{3} \pi}-\frac{(\mu-1)(7 \mu-31)}{3 \cdot 2^{7} \pi^{3}(n+m)^{2}}-\frac{(\mu-1)\left(83 \mu^{2}-982 \mu+3779\right)}{15 \cdot 2^{10} \pi^{5}(n+m)^{4}} \\
& -\frac{(\mu-1)\left(6949 \mu^{3}-153855 \mu^{2}+1585743 \mu-6277237\right)}{105 \cdot 2^{15} \cdot \pi^{7} \cdot(n+m)^{6}} \\
& -\frac{(\mu-1)\left(70197 \mu^{4}-2479316 \mu^{3}+48010494 \mu^{2}\right.}{40320 \cdot 2^{11} \cdot \pi^{9}(n+m)^{8}}
\end{aligned}
$$

Suppose that the problem is to ('alculate the 14th zero of $J_{5 / 2}(z)$ or $z_{5 / 2}^{(14)}$. Then $m=1$, and we should want to find $\bar{S}_{15}$ using Table 4 upon $\bar{S}_{4}-\bar{S}_{10}$, after which we obtain $\bar{z}_{5 / 2}^{(14)}$ from $(13) .^{* *}$ From $(14)$ and then $(13), \bar{z}_{5 / 2}^{(14)}$ which is equal to $z_{5 / 2}^{(14)}$ to around $14 \mathrm{D}$, is found to be 47.06014161276054 . A quick examination of the

\begin{tabular}{|c|c|}
\hline & $\bar{s}_{i}$ \\
\hline & -0.9737185140725358 \\
\hline & $-0.96680 \quad 12788 \quad 752868$ \\
\hline 6 & -0.96331173960268038 \\
\hline 7 & $-0.9609204667 \quad 126258$ \\
\hline & $-0.95950+2113725122$ \\
\hline 9 & $\begin{array}{llll}-0.95853 & 75688 & 13022 & 8\end{array}$ \\
\hline 10 & $-0.9578+828+501+485$ \\
\hline
\end{tabular}
ratios of successive terms in (14) indicates without having to compute the $1 /(n+m)^{10}$ term that, to $14 \mathrm{D}, z_{5 / 2}^{(14)}$ is actually 47.06014161276053 . Following are the calculated values of $\bar{S}_{j}$, for $j=n+1=4(1) 10$, to $16 \mathrm{D}$ (last figure approximate):

Employing the older $(1 / j)$-extrapolation, we find $\bar{S}_{15}=-0.9562228677507 \ldots$ and from $(13), \bar{z}_{5 / 2}^{(14)}=\$ 7.06014161266 \ldots$ which agrees with the true value of

${ }^{*}$ The coefficients through $1 /(n+m)^{6}$ are from Watson [11], and the coefficient of $1 /(n+m)^{8}$ is from Bickley and Miller [13].

** This particular problem could, of course, be set up equally efficiently computationwise by writing $\bar{S}_{n+1}=a_{0}+a_{1} /(n+1)^{2}+\cdots+a_{r} /(n+1)^{2 r}$, where $a_{i}$ is independent of $n$. But this present method works as long as we know somehow the values of $\bar{S}_{j}$. 
$z_{\mathrm{B} / 2}^{(14)}$ to a unit in the 10th decimal (12th significant figure). But the $\left(1 / j^{2}\right)$-extrapolation yields $\bar{S}_{1 \mathrm{~s}}=-0.95622286629517 \ldots$ and from $(13), \bar{z}_{5 / 2}^{(14)}=47.06014$ $161276055 \ldots$ which almost agrees with the true value of $z_{5 / 2}^{(14)}$ to 14 decimals (16 significant figures).

7. Acknowledgment. Most of the checking of the tables and the checking of all the examples was done by Mrs. Elizabeth P. Thompson, to whom the authors wish to express their appreciation.

\section{Convair-Astronautics}

San Diego, California

1. H. E. SALZER, "A simple method for summing certain slowly convergent series," $J$. Math. Phys., v. 33, 1955, p. 356-359.

2. H. E. SALzER, "Formulas for the partial summation of series," $M T A C$, v. 10, 1956, p. 149-156.

3. L. F. Richardon, "The approximate arithmetical solution by finite differences of physical problems involving differential equations, with an application to the stresses in a masonry dam," Philos. Trans. Roy. Soc. London, Ser. A, v. 210, 1910, p. 307-357.

4. L. F. Richandson \& J. A. GaUnT, "The deferred approach to the limit," Philos. Trans. Roy. Soc. London, Ser. A, v. 226, 1927, p. 299-361.

5. G. Buance, "On the numerical solution of parabolic partial differential equations," J. Res. Nat. Bur. Standards, v. 50, 1953, p. 343-356.

6. H. C. Bolton \& H. I. Scorns, "Eigenvalues of differential equations by finite-difference methods," Proc. Cambridge Philos. Soc., v. 52, pt. 2, 1956, p. 215-229.

7. M. G. Salvadori, "Extrapolation formulas in linear difference operators," Proc. First U. S. Nat. Congress of Applied Mech., Edwards Bros., 1952, p. 15-18.

8. Herrmann, "Bestimmung der trigonometrischen Functionen aus den Winkeln und der Winkel aus den Functionen, bis zu einer beliebigen Grenze der Genauigkeit," Kaiserliche Akademie der Wissenschaften, Wien, Mathematisch-naturwissenschaftliche Classe, Sitzungsberichte, 1, pt. IV, 1848, p. 176-177. Reprinted in Table of Sines and Cosines to Fifteen Decimal Places at Hundredths of a Degree, Nat. Bur. Standards. A ppl. Math. Ser. No. 5, 1949, p. 92-93.

9. E. WhitTaker \& G. Robinson, The Calculus of Observations, 4 th edition, Blackie and Son, London, 1954, p. 135.

10. H. T. DAvis, Tables of the Higher Mathematical Functions, v. II, Principia Press, Bloomington, Indiana, 1935, p. 282, 284-285, 304. Davis cites the earlier work of J. W. L. Glaisher in relation to Catalan's constant in Mess. of Math., v. 6, 1876, p. 71-76, Proc. London Math. Soc., v. 8, 1877, p. 200-201, Mess. of Math., v. 42, 1913, p. 35-58.

11. G. N. Watson, Theory of Bessel Functions, 2nd edition, Cambridge University Press, 1952 , p. 506

12. NBS, Tables of Spherical Bessel Functions, v. II, New York, Columbia University Press, 1947, p. 318.

13. W. G. BICKLEY \& J. C. P. MILLER, "Notes on the evaluation of zeros and turning values of Bessel functions, II, The McMahon Series," Phil. Mag., s. 7, v. 36, 1945, p. 125. 